\title{
Changes in blood parameters induced by experimental jaagsiekte sheep retrovirus infection
}

\author{
Katarzyna Dudek ${ }^{1}$, Krzysztof Lutnicki ${ }^{2}$, Dariusz Bednarek ${ }^{1}$, \\ Jan Marczuk ${ }^{2}$, Anna Kycko ${ }^{3}$, Michał Reichert ${ }^{3}$ \\ ${ }^{1}$ Department of Cattle and Sheep Diseases, \\ ${ }^{3}$ Department of Pathology, \\ National Veterinary Research Institute, 24-100 Pulawy, Poland \\ ${ }^{2}$ Sub-Department of Internal Diseases of Farm Animals and Horses, \\ Department and Clinic of Animal Internal Diseases, Faculty of Veterinary Medicine, \\ University of Life Sciences in Lublin, 20-612 Lublin, Poland \\ katarzyna.dudek@piwet.pulawy.pl
}

Received: June 3, 2016

Accepted: September 6, 2016

\begin{abstract}
The aim of the study was to assess the changes of blood parameters in 12 three-week-old Polish Merino sheep subjected to experimental jaagsiekte sheep retrovirus (JSRV) infection. Material and Methods: Haematological (WBC with leukocyte subpopulations: GRA, LYM, MID, and RBC, MCV, MCH, MCHC, HGB, HCT, PLT, and MPV) and biochemical blood parameters (acid/base balance, cation/anion content, and gasometry) were determined in blood samples collected one month after JSRV infection, then at four-week intervals for five consecutive months. Results: A decrease in RBC, HCT, MCV, PLT, MPV, and LYM values in comparison with controls was found in the last month of observation. On the other hand, at the same time, an increase in $\mathrm{HGB}, \mathrm{MCH}, \mathrm{MCHC}, \mathrm{WBC}, \mathrm{MID}$, and GRA indices was observed. Moreover, at the end of experiment blood gasometric indices such as $\mathrm{pCO}_{2}, \mathrm{HCO}_{3}$, and $\mathrm{tCO}_{2}$, and $\mathrm{Na}$ and $\mathrm{K}$ ion concentrations were higher in the affected lambs than in the healthy animals. The $\mathrm{pH}$ values of the challenged animals exhibited less alkaline character than in the case of controls, which was associated with a decrease in $\mathrm{O}_{2} \%$ saturation. However, the majority of differences between JSRV inoculated and control groups was not statistically significant. Conclusion: The observed changes in the examined blood parameters can be considered as prodromal symptoms in the preclinical phase of adenocarcinoma development associated with JSRV infection.
\end{abstract}

Keywords: sheep, jaagsiekte sheep retrovirus, adenocarcinoma, haematological and biochemical parameters.

\section{Introduction}

Ovine pulmonary adenocarcinoma (OPA) is a contagious tumour of sheep originating from the pneumocytes type II and bronchiolar Clara cells, associated with pulmonary infection caused by jaagsiekte sheep retrovirus (JSRV). The virus belongs to Retroviridae family, Orthoretrovirinae subfamily, and the genus of Betaretrovirus. Viral genome is composed of linear, positive, single-stranded RNA, and it comprises four main genes organised as 5' - gag- propol- env -3' which encode the virus proteins. The integrated JSRV genome is flanked by the long terminal repeat (LTR) which is the site of transcriptional initiation activated in the secretory epithelial cells in ovine lungs. The gene env encodes viral envelope protein which is a major determinant of the transformation of differentiated lung epithelial cells: type II pneumocytes in the alveoli and Clara cells in the bronchioles $(8,9,11)$. The disease, also called ovine pulmonary adenomatosis (OPA) or jaagsiekte, is present worldwide, except for Australia and New Zealand, and it has been eradicated from Iceland. In Poland, suspicion of its occurrence was last reported in 2002 (16). OPA has a long incubation period, which takes at least three weeks in experimentally infected lambs and usually over two years in adult sheep before clinical signs of respiratory tract distress can be observed. Rapid breathing reflects the extent of tumour development in the lungs. Raising the back and 
lowering the head of the sheep may cause a nasal discharge of frothy mucoid fluid accumulated within respiratory tract (12).

To date, diagnosis of the disease has been possible only when clinical signs became apparent and not during the preclinical period of the disease. There is no serological test for OPA due to the presence of endogenous viral sequences in sheep genome which cause lack of circulating JSRV specific antibodies, as has been demonstrated by Western blotting $(7,12)$.

At present, diagnosis relies on clinical history and clinical signs which have to be confirmed by necropsy and histopathology, or by PCR-based methods allowing the detection of JSRV in infected animals $(3-6,14)$. The virus induces neoplastic transformation of secretory epithelial cells of the lung forming a tumour which can be considered as a good model for investigation of human lung cancer oncogenesis and therapy.

The aim of this study was to evaluate the blood morphological and biochemical parameters in the course of OPA and assess them as prodromal markers of tumour development.

\section{Material and Methods}

Sample collection. Twelve Polish Merino lambs, three-week-old, were used in the experiment. The lambs were kept in stalls together with their mothers. Initially they were nursed by their mothers and fed colostrum. About five weeks after birth they gradually started to eat hay, grass and grain. Six lambs were experimentally infected by intratracheal inoculation with JSRV clone (kindly provided by Prof. M. Palmarini, University of Glasgow, Scotland) (9). The remaining animals served as control. Blood samples were collected from the jugular vein of all animals one month after JSRV infection of the experimental lambs, then at four-week intervals for five consecutive months. All the infected lambs were euthanised using intravenous injection of pentobarbital sodium (140 mg/kg b.w.) after developing clinically visible signs of the disease, i.e. dyspnea and weakness. After the experiment control animals also underwent euthanasia in the same way as the infected ones.

Haematological analyses. The blood samples for determination of haematological parameters were collected into tubes containing anticoagulant, and analyses were performed ex tempore. The following indices were analysed: white blood cell (WBC) count with leukocyte subpopulations such as polymorphonuclear leukocytes (GRA), lymphocytes (LYM), monocytes, eosinophils, and basophils as midsize cells (MID), red blood cell (RBC) count, mean corpuscular volume (MCV), mean corpuscular haemoglobin $(\mathrm{MCH})$, mean corpuscular haemoglobin concentration (MCHC), mean erythrocyte haemoglobin (HGB), haematocrit (HCT), platelet count (PLT), and mean platelet volume (MPV). Haematological parameters were measured using an automatic veterinary blood analyser (Exigo, Boule Medical AB, Sweden).

Biochemical analyses. The following parameters were determined: acid/base balance as $\mathrm{pH}$, bicarbonate $\left(\mathrm{HCO}_{3}\right)$, lactic acid (LA), total haemoglobin ( $\left.\mathrm{tHb}\right)$, cation/anion content $\left(\mathrm{Na}^{+}, \mathrm{K}^{+}, \mathrm{Cl}^{-}\right)$, partial pressure of carbon dioxide $\left(\mathrm{pCO}_{2}\right)$, total carbon dioxide $\left(\mathrm{tCO}_{2}\right)$, partial pressure of oxygen $\left(\mathrm{pO}_{2}\right)$, oxygen saturation of haemoglobin $\left(\mathrm{sO}_{2}\right)$ using IDEXX Vet Stat Electrolyte and Blood Gas Analyzer, USA.

Clinical signs. The animals were observed for the occurrence of visible signs of lung tumour development, such as dyspnea, weakness, discharge of frothy exudate from nostrils.

Gross pathology. All the euthanised animals were submitted to necropsy for evaluation of OPA-related changes in the lungs evidenced by neoplastic nodules and fluid in the pulmonary airways.

Histological examination. During necropsy, lung tissue samples were collected for histological examination from four parts of the lungs. The samples were fixed in $10 \%$ neutral buffered formalin, processed routinely in an automatic tissue processor, embedded in paraffin, and sectioned into 4 - to $6-\mu \mathrm{m}$-thick sections. The sections were stained with haematoxylin and eosin and examined under light microscopy for the presence of tumour lesions.

DNA isolation and PCR. Lung tissue samples of the animals were collected for detection of viral DNA using PCR method. Frozen lung tissue samples of OPA-affected sheep kindly provided by Prof. M. De Las Heras (University of Zaragoza, Spain) and archival frozen lung tissue samples of non-affected sheep were used for DNA isolation and then served as a positive and negative control for the PCR. DNA was isolated from $30 \mathrm{mg}$ of each collected lung tissue sample using Genomic Mini Kit (A\&A Biotechnology, Poland), according to manufacturer's recommendations. The PCR was conducted as previously described (6).

Statistical analysis. Statistical significance of differences between both animal groups, i.e. JSRVinoculated and control, was calculated with the MannWhitney $U$ test. The differences were considered significant at $\mathrm{P}<0.05$. Calculations were performed using Statistica v. 10.0 PL.

\section{Results}

Clinical signs, gross pathology, and histopathological findings. The JSRV-inoculated lambs showed clinical signs of progressive respiratory illness, i.e. rapid breathing associated with noticeable movement of the abdominal wall. In each case lowering of the head caused a discharge of mucoid fluid from the nostrils. Necropsy revealed multifocal to coalescent grey, nodular, firm, hyperplastic lesions in 
the lungs (Fig. 1A), accompanied by the presence of frothy white fluid in the respiratory passages, flowing out of the trachea when it was cut (Figs 1B and 1C). Histological examination revealed that the nodules were composed of single to multiple layers of cuboidal or elongated cells lining alveolar walls and frequently forming papillary projections. These lesions were characteristic of pulmonary adenocarcinoma (Fig. 2). Infiltration of inflammatory cells, mostly plasmocytes, was also observed in the lung parenchyma.

PCR. The PCR analysis (Fig. 3) confirmed the presence of JSRV specific sequences in all tumour tissues.

Haematological and biochemical analyses. The analysis of the white blood cell populations indicated that after the inoculation the WBC count was visibly increased in the first, second, and fifth month when compared to control. In contrast, it had lower values in months three and four in the inoculated lambs. The GRA percentage increased after the infection throughout the study. At the same time, contrary to the previously described parameter, the LYM percentage decreased in the inoculated animals when compared to controls.

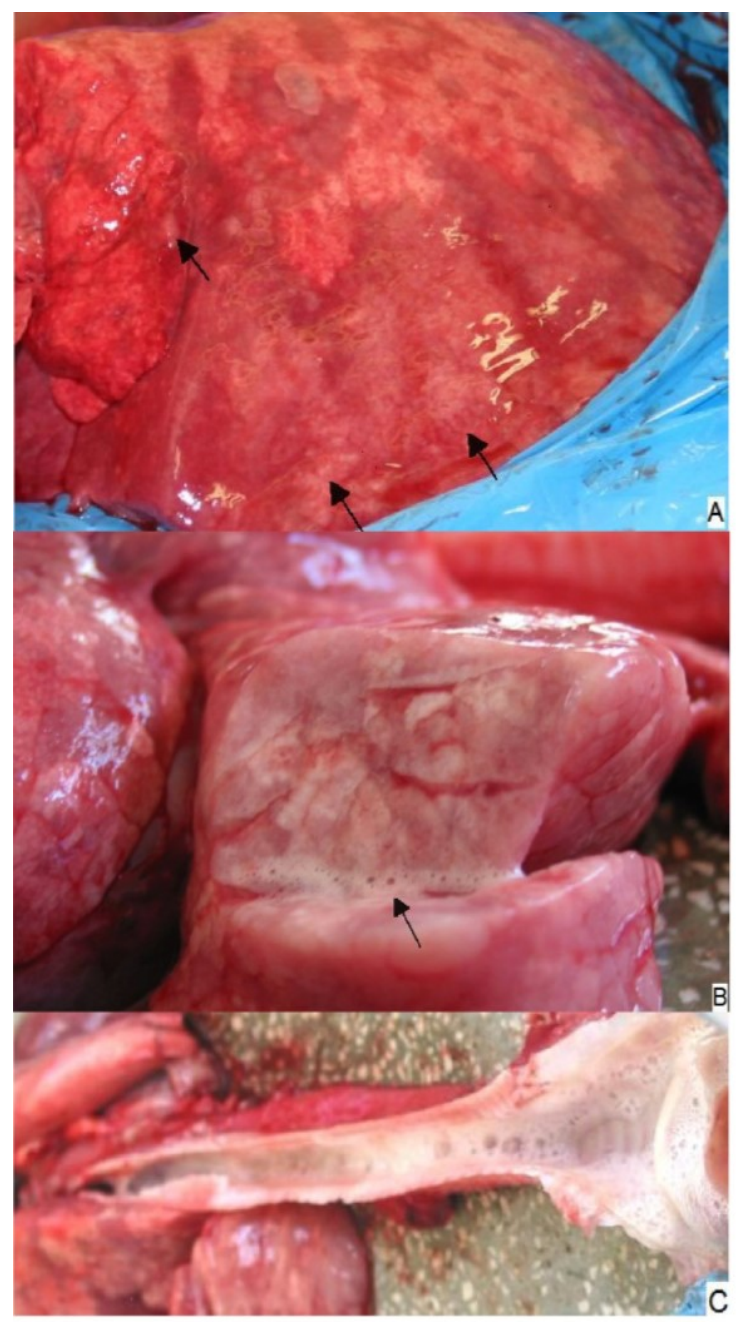

Fig. 1. Lungs of JSRV-infected sheep. (A) Grey nodular lesions in the lungs (arrows), (B) pale frothy fluid pouring from the bronchioli on a cut surface of the lungs (arrow), (C) frothy fluid in the trachea

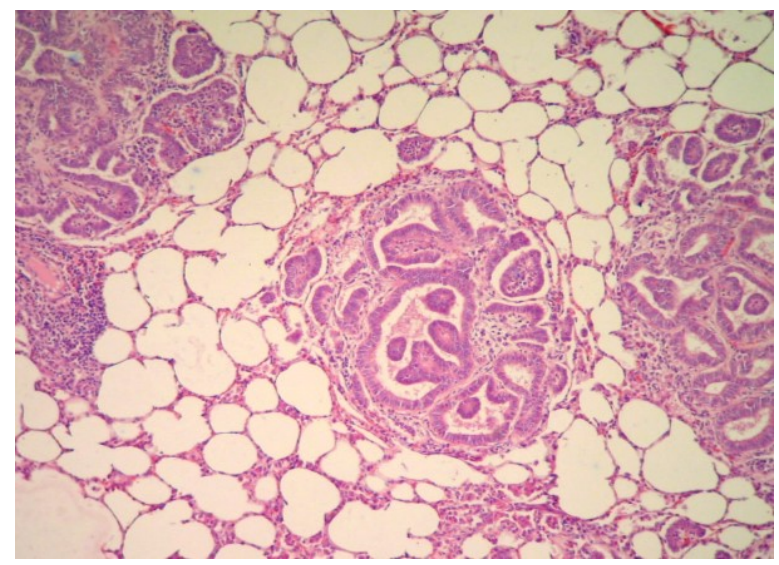

Fig. 2. Ovine pulmonary adenocarcinoma. Adenomatous lesions are formed by neoplastic cells deriving from pulmonary secretory epithelium. HE, 100×

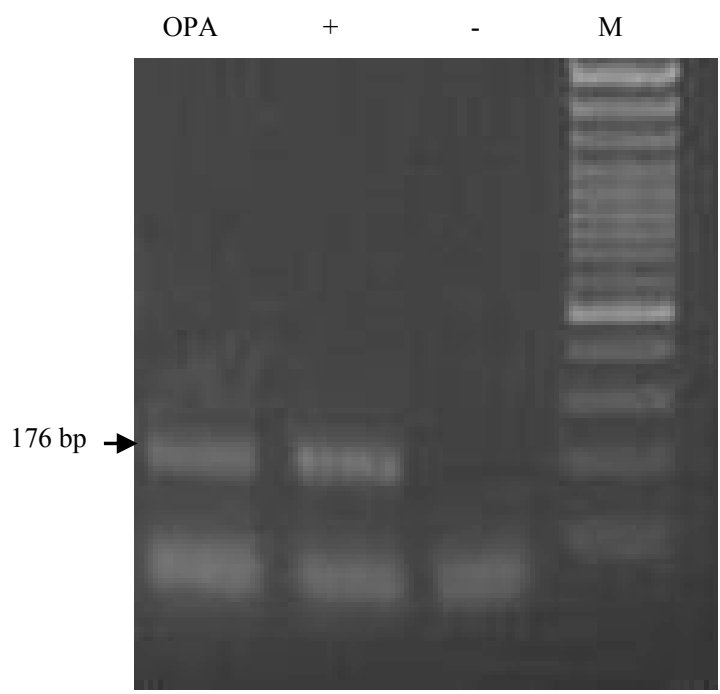

Fig. 3. Agar gel electrophoresis of PCR reaction products for JSRV presence in DNA isolated from lung tumour of one of the sheep inoculated with JSRV (OPA). + (positive control), - (negative control), M (molecular weight marker), 176bp (a length of the product)

After the inoculation the MID percentage increased in the first, third, and fifth month, whereas during the remaining months it was slightly lower in the inoculated lambs. The haematological examination revealed that the RBC count significantly decreased one month after the JSRV infection. However, during the next two months it was visibly higher than the control values. Finally, in the last month of the study, the RBC count was significantly reduced again in the JSRV inoculated lambs. The differences between the two groups were statistically significant in the first, second, third, and fifth month after the JSRV infection. During the first two months post inoculation, the MCV was higher than in control. Otherwise, during the next three months of the study it decreased. The $\mathrm{MCH}$ decreased during the first three months of the study when compared to control, whereas in months four and five it was significantly increased in the JSRVinoculated animals. Between months one and three the $\mathrm{MCHC}$ values were lower in the inoculated lambs than 
in controls. After that they began to increase until the end of the study. The HGB increased in the inoculated lambs throughout the study when compared to control, especially during the first two months. In months one, four, and five after the inoculation, the HCT values were lower than in control, whereas in the remaining months they increased in the infected animals. During the first three months after the infection, the PLT increased in comparison with control. In contrast, in months four and five it decreased in the inoculated lambs. On the other hand, in the case of MPV changes, with the exception of month two, the values were lower than in control throughout the study (Fig. 4).

The biochemical analysis showed a decrease in $\mathrm{pH}$ values in the inoculated lambs during the study, with the exception of month one (Fig. 5).
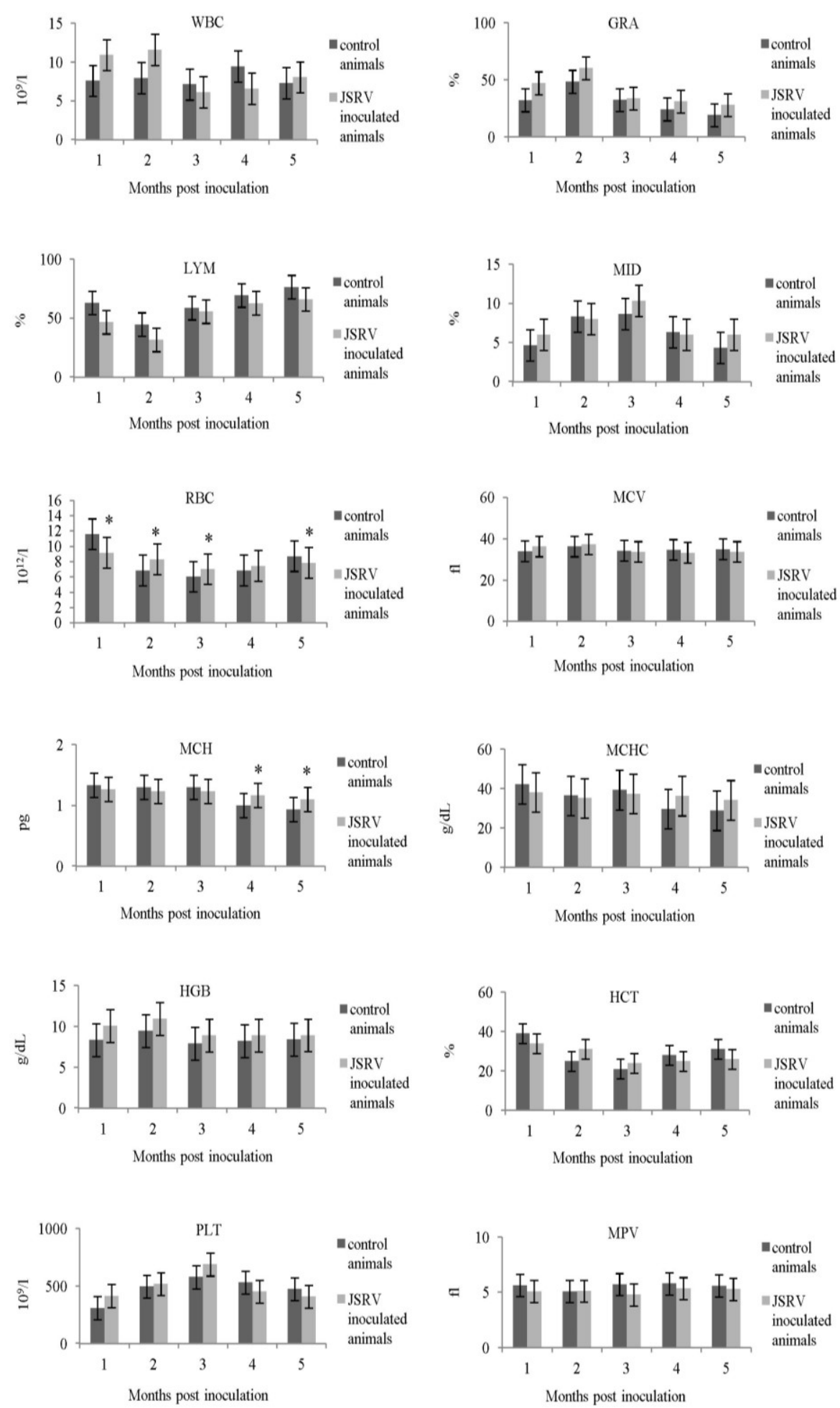

Fig. 4. Changes in the selected haematological parameters (WBC, GRA, LYM, MID, RBC, MCV, MCH, MCHC, HGB, HCT, PLT, MPV) in the blood of the JSRV-inoculated and control lambs. * $\mathrm{P}<0.05$ between groups. The values are presented as mean $\pm \mathrm{SD}$ 

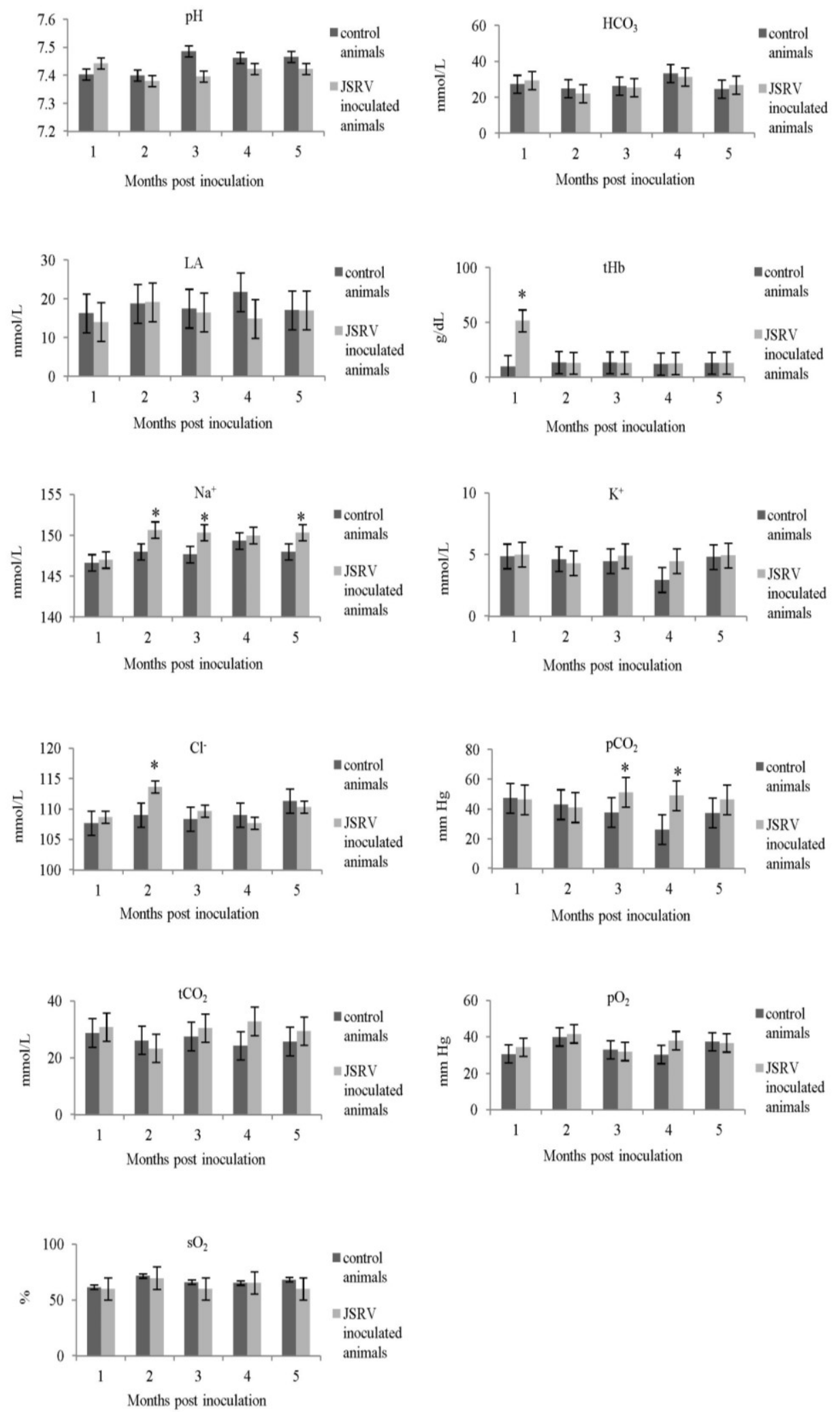

Fig. 5. Changes in the selected biochemical parameters ( $\mathrm{pH}, \mathrm{HCO}_{3}$, $\mathrm{LA}, \mathrm{tHb}, \mathrm{Na}^{+}, \mathrm{K}^{+}, \mathrm{Cl}^{-}, \mathrm{pCO}_{2}, \mathrm{tCO}_{2}, \mathrm{pO}_{2}, \mathrm{sO}_{2}$ ) in the blood of the JSRV-inoculated and control lambs. ${ }^{*} \mathrm{P}<0.05$ between groups. The values are presented as mean \pm SD

Similarly, the $\mathrm{HCO}_{3}$ values decreased in the inoculated lambs between months two and four, whereas in the first and fifth month they were higher when compared with the control values. After the inoculation the LA decreased in the first, third, and fourth month, whereas in the remaining months it was close to the control 
values. The tHb significantly increased in the inoculated lambs in month one when compared to control, whereas during the next months it was close to the control values. The $\mathrm{Na}^{+}$values were generally higher in the inoculated lambs throughout the study in comparison with controls. The statistically significant differences within the parameter between the animal groups were observed in the second, third, and fifth month of the study. After the experimental infection, the $\mathrm{K}^{+}$values visibly increased in months three and four. In contrast, in month two they were slightly decreased than in control, whereas during the remaining months the $\mathrm{K}^{+}$values were close to control with increasing tendency. During the first three months, the $\mathrm{Cl}^{-}$values were higher in the inoculated lambs than in controls, especially in month two when a statistically significant difference between the two animal groups was shown. In contrast, during the remaining months of the study these values were lower than in controls. After the infection, the $\mathrm{pCO}_{2}$ was visibly higher than in control between months three and five (significantly in the third and fourth month), whereas in the first two months it was slightly decreased when compared to control. With the exception of month two, the $\mathrm{tCO}_{2}$ was increased in the inoculated animals throughout the study in comparison with controls. By contrast, after the inoculation the $\mathrm{pO}_{2}$ increased in months one and four when compared to control, whereas in the remaining months it was close to the control values. A slight decrease in the $\mathrm{sO}_{2}$ values was observed in the inoculated lambs in months three and five when compared to control. In the remaining months the $\mathrm{sO}_{2}$ values were similar in both groups (Fig. 5).

In spite of visible differences in the analysed parameters between JSRV-inoculated and control groups, especially these significantly changed like the $\mathrm{RBC}, \mathrm{MCH}, \mathrm{tHb}, \mathrm{Na}^{+}, \mathrm{Cl}^{-}, \mathrm{pCO}_{2}$, the majority of them were not statistically significant.

\section{Discussion}

In contrast to the reports on the significance of pathological and molecular methods for diagnosis of OPA, alterations of haematological and biochemical parameters have not been sufficiently addressed in the literature so far $(2,12)$. The present study fills this gap and provides new and interesting data. The results have shown visible changes of basic haematological parameters as well as biochemical ones; however, not all of them were statistically significant. The individual dynamics of these parameters was different.

The RBC count below the physiological values observed in months one and five after the inoculation may indicate the beginning of anaemia. However, this status has been quickly compensated and in the remaining months it was in the physiological range for the species of breeding animals. The generally low HCT during the infection is typical for the cases of body overhydration and hypervolemia as well as anaemia. Low values of HCT were also reported in the cases of increased volume of circulating blood, as a result of nephrosis, late pregnancy, body overhydration, or during viral and bacterial infections $(1,10,13)$. Haemolytic anaemia also results in a low HCT (15). The changes in the MCV noted in our experiment resulted in microcytosis, which is typical for hypertonic overhydration, and in combination with low values of the RBC, HGB, and HCT are the basis for diagnosis of microcytic anaemia (17). Additionally, a slight increase in the MCV during the first months after the infection could be caused by the appearance of an increased number of reticulocytes. The thrombocytosis observed in the first period of the JSRV infection may suggest an activation of blood coagulation process, whereas permanent lymphopenia is typical for viral infection. On the other hand, the leukocytosis observed in the first two months after the inoculation and in month five resulted from visible granulocytosis, which probably indicates increased synthesis and mobilisation of polymorphonuclear cells after the infection.

The results of the study indicated that neoplastic process in the lungs of experimental animals caused the changes in haematological as well as biochemical parameters. The observed changes were rather slight but in some cases statistically significant. The changes in the $\mathrm{pCO}_{2}$ after the infection indicated a progressive alkalosis. Other biochemical changes enhanced ventilation (hyperpnoea) dependent on the change of the acidic to more basic values of $\mathrm{pH}$ and an increase in the oxygen saturation and the initiation of compensatory and corrective response with still efficient mechanisms of acid-base balance in the first period of the disease. Therefore, the observed acid-base disturbances point to a progressive disorder of the respiratory system manifested by the increased blood flow resistance and impaired gas exchange in the lungs and secondary cardiac failure at the same time.

The results can be useful in veterinary practice, because described changes in the blood parameters occurred in the OPA affected animals significantly earlier than the clinical (respiratory) symptoms. Therefore, determination of these parameters, although not pathognomonic, can be helpful in the early diagnosis of OPA.

Conflict of Interests Statement: The authors declare that there is no conflict of interests regarding the publication of this article.

Financial Disclosure Statement: This study was financially supported by the Polish National Science Centre Project no.: N N308 256335, contract no.: PB 2563/B/P01/2008/353.

Animal Rights Statement: The authors declare that the experiments on animals were conducted in 
accordance with local Ethical Committee laws and regulations as regards care and use of laboratory animals $\left(2^{\text {nd }}\right.$ Local Ethical Committee for Animal Experiments in Lublin).

Acknowledgements: The authors thank Professor Massimo Palmarini, University of Glasgow, Scotland for providing JSRV clone.

\section{References}

1. Bouzar A.B., Boxus M., Florins A., François C., Reichert M., Willems L.: Reduced levels of reactive oxygen species correlate with inhibition of apoptosis, rise in thioredoxin expression and increased bovine leukemia virus proviral loads. Retrovirology 2009, 6, 102.

2. Cousens C., Scott P.R.: Assessment of transthoracic ultrasound diagnosis of ovine pulmonary adenocarcinoma in adult sheep. Vet Rec 2015, 177, 366.

3. De Las Heras M., Ortín A., Salvatori D., Pérez de Villareal M., Cousens C., Miguel Ferrer L., Miguel Cebrián L., García de Jalón J.A., Gonzalez L., Michael Sharp J.: A PCR technique for the detection of jaagsiekte sheep retrovirus in the blood suitable for the screening of ovine pulmonary adenocarcinoma in field conditions. Res Vet Sci 2005, 79, 259-264.

4. González L., García-Goti M., Cousens C., Dewar P., Cortabarría N., Extramiana A.B., Ortín A., De las Heras M., Sharp J.M.: Jaagsiekte sheep retrovirus can be detected in the peripheral blood during the pre-clinical period of sheep pulmonary adenomatosis. J Gen Virol 2001, 82, 1355-1358.

5. Holland M.J., Palmarini M., García-Goti M., González L., McKendrick I., De las Heras M., Sharp J.M.: Jaagsiekte retrovirus is widely distributed both in $\mathrm{T}$ and $\mathrm{B}$ lymphocytes and in mononuclear phagocytes of sheep with naturally and experimentally acquired pulmonary adenomatosis. J Virol 1999 , 73, 4004-4008.

6. Kycko A., Reichert M.: PCR-based methods for detection of JSRV in experimentally and naturally infected sheep. Bull Vet Inst Pulawy 2010, 54, 445-450.
7. Ortín A., Minguijón E., Dewar P., García M., Palmarini M., González L., Sharp J. M., De las Heras M.: Lack of specific immunoresponse against recombinant capsid antigen of the sheep pulmonary adenomatosis retrovirus in sheep and goats naturally affected by enzootic intranasal tumour or jaagsiekte. Vet Immunol Immunopathol 1998, 61, 229-237.

8. Palmarini M., Fan H.: Molecular biology of jaagsiekte sheep retrovirus. Curr Top Microbiol Immunol 2003, 275, 81-115.

9. Palmarini M., Sharp J.M., De las Heras M., Fan, H.: Jaagsiekte sheep retrovirus is necessary and sufficient to induce a contagious lung cancer of sheep. J Virol 1999, 73, 6964-6972.

10. Rehulka J.: Haematological analyses in rainbow trout Oncorhynchus mykiss affected by viral haemorrhagic septicaemia (VHS). Dis Aquat Organ 2003, 56, 185-193.

11. Salvatori D., González L., Dewar P., Cousens C., de las Heras M., Dalziel R.G., Sharp J.M.: Successful induction of ovine pulmonary adenocarcinoma in lambs of different ages and detection of viraemia during the preclinical period. J Gen Virol 2004, 85, 3319-3324.

12. Scott P., Griffiths D., Cousens C.: Clinical practice - farm animals: Diagnosis and control of ovine pulmonary adenocarcinoma (jaagsiekte). In Practice 2013, 35, 382-397.

13. Van Amstel S.R., Reyers F., Myburgh E., Pretorius G., Sacks P.: The clinical pathology of heartwater. III. Changes in blood clotting, blood calcium, blood protein, haematocrit and whitecell counts in sheep with experimentally induced heartwater. Onderstepoort J Vet Res 1994, 61, 21-27.

14. Voigt K., Brügmann M., Huber K., Dewar P., Cousens C., Hall M., Sharp J.M., Ganter M.: PCR examination of bronchoalveolar lavage samples is a useful tool in pre-clinical diagnosis of ovine pulmonary adenocarcinoma (jaagsiekte). Res Vet Sci 2007, 83, 419-427.

15. Wolf R.B., Moise K.J. Jr, Brace R.A.: Antibody-induced anemia in fetal sheep: model for hemolytic disease of the fetus and newborn. J Soc Gynecol Investig 2001, 8, 224-232.

16. World Organization for Animal Health, Handistatus II Multiannual Animal Disease Status 2006. https://web.oie.int/hs2/report.asp.

17. Yuan-Rong, li-Juan L., Qi-Wen W., Guo-Zhen D.: Copper deficiency in Guizhou semi-fine wool sheep on pasture in south west China karst mountain area. Afr J Biotechnol 2011, 10, 17043-17048. 